\title{
Beitrag zur Kenntniss des Athmungscentrums.
}

$$
\text { Von }
$$

\section{Dr. Richard Nitschmann.}

In den Comptes rendus vom Jahre 1847-1851 verlegt Flourens das Athmungscentrum in die hintere Spitze des calamus scriptorius und nennt diesen Punkt wegen der Wichtigkeit seiner Erhaltung für das Leben gleichsam als Lebenscentrum noeud vital oder point vital.

Im Laufe der Jahre ist diese Floure ns'sche Ansicht von den verschiedensten Autoren bekämpft und dem noeud vital allmälig immer mehr und mehr von seiner Wichtigkeit geraubt worden. Die Arbeiten die hieruber vorliegen lassen sich füglich in zwei Hauptgruppen zerlegen, nämlich die einen belassen das Athmungscentrum dem verlängerten Marke und suchen es nur dem Flourens'schen noeud vital zu entreissen, die anderen weisen nach, dass das in der medulla oblongata enthaltene Athmungscentrum nicht das einzige sei, sondern dass im Cervicalmarke noch Nervencentren bestehen müssten, die auf die Athmung von bedeutendem Einflusse seien.

Was die erste Reihe von Arbeiten anbetrifft, so hat BrownSéquard ${ }^{1}$ ) nachgewiesen, dass man mit einiger Vorsicht den noeud vital entfernen oder zerstören kann, ohne dass das Thier nothwendig zu athmen aufhöre. Das Gleiche fand auch Sehiff ${ }^{2}$ ), und dieser letzte Autor verlegt das Athmungscentrum auf beide Seiten des verlängerten Markes etwas hinter die Austrittsstelle der Vaguswurzeln in die Gegend des vorderen Theiles der alae cinereae.

1) Researches on the spinal chord. Richmond 1855, und Recherches sur les causes de mort après l'ablation de la partie de la moelle allongée, qui a été nommé noeud vital. Journal de la Physiologie 1858.

2) Lehrbuch der Physiologie des Mensehen I, Muskel und Nervenphysiologie $1858-1859$. 
Longet ${ }^{1}$ ) und Volkmann ${ }^{2}$ ) spalteten den noend vital durch einen Längsschnitt, ohne dass die Athmung der Thiere unregelmässig geworden wäre oder aufgehört hätte.

Gielrk $\mathrm{e}^{3}$ ) fand, dass Verletzung oder Zerstörung der sogenannten Respirationsbündel ${ }^{4}$ ) Athmungsstillstand erzeuge und zwar einseitige Verletzung auch nur einseitigen Stillstand auf gleichnamiger Körperhälfte und nur Verletzung beider Respirationsbündel vollständiges Aufhören der Athmụng. Eine Zellengruppe, der er den Namen Athmungscentrum hätte beilegen können hat er bei seinen genauen and ausfubrlichen mikroskopischen Untersuchungen nicht mit Sicherheit gefunden.

Zu der zweiten Gruppe von Arbeiten hat zunächst auch wieder B rown-Séquard ${ }^{5}$ ) Beiträge geliefert, der bereits im Jahre 1860 den Satz aussprach: „l'ablation de la moelle allongée n'amène pas nécessairement la cessation immédiate des mouvements respiratoires." Ihm folgen dann einige Jahre später 1874 Rokitansky ${ }^{6}$ ), welcher sagt, dass Kaninchen mit durchtrenntem Halsmarke, wenn sie mit Strychnin vergiftet werden, noch Athembewegungen ausführen, und 1875 Schroff 7 ), welcher nach Durchtrennung des Halsmarkes auch bei nicht strychninisirten Thieren spontane Athmungen sieht, wenn dieselben in einen Wärmekasten gelegt werden und ihnen längere Zeit künstliche Athmung zugeführt wird, welche letztere dann plötzlich auf gewisse Zeitdauer unterbrochen wird. Doch hat Schroff meist nur 2-3 Athemzüge gesehen, und sagt selbst es scheine ihm die Kraft der spinalen Athmungscentren sehr leicht erschöpft zu sein. Aus neuerer Zeit sind in der Literatur iiber dieses Thema erschienen, einmal eine Arbeit von Lauten-

1) Expériences relatives aux effets de l'inhalation de l'éther sulfurique sur le système nerveux de l'homme et des animaux. Archives générales de méd. 1847, Tome XIII, pag. 377.

2) Wagner, Handwörterbuch der Physiologie. Artikel: Gehirn.

3) Dies Archiv Bd. VII, S. 583.

4) Nach Schwalbe stellt dasselbe eine im Halsmarke entstehende, aufsteigende Wurzel der Vagusgruppe vor. Hoffmann-Schwalbe, Lehrbuch der Anatomie des Menschen. II. Auflage, S. 663.

5) Journal de la physiologie de l'homme et des animaux. Paris. Tom. III. pag. 153 .

6) Wiener medizinische Jahrbücher. Jahrgang 1874, S. 33.

7) Wiener medizinische Jabrbücher. Jahrgang 1875, S. 324. 
ba ch'1), die mir leider nicht zugänglich war, sodass ich sie nur anführen kann ohne genaueres ïber ihren Inhalt zu kennen, und mehrere Veröffentlichungen von Langendorff $f^{2}$ ), bei deren vorbereitenden Versuchen ich zum grossen Theil dabei sein durfte, und deren einen Theil mir auch selbst auszuführen gestattet war. Da meine jetzige Arbeit sich enge an die Langendorff'schen Mittheilungen anschliesst, und $d a$ ich bei denselben zum Theil mit interessirt bin, wie ja auch Herr Dr. Langendorff so freundlich gewesen ist die erste Mittheilung ${ }^{3}$ ) in seinem und meinem Namen zu veröffentlichen, so sei es mir gestattet mit einigen Worten auf dieselben zuriickzukommen.

Ende des Jahres 1879 wurden die von Volkmann4) und Longet ${ }^{4}$ ) angegebenen Versuche iiber die langitudinale Durchstechung der Spitze des calamus scriptorius, ohne dass die Athmung still steht, wiederholt und bestätigt. Dabei wurde aber ferner gefunden, dass nach der Vagotomie oder auch bei intacten vagi auf einseitigen Vagusreiz oder einseitigem Trigeminusreiz die Athmung entweder, je nach der Intensität des Reizes, einseitig - auf gleicher Körperhälfte - stille steht oder nur verlangsamt wird, wogegen Reize des brachialis oder des ischiadicus exspiratorischen Stillstand auf beiden Seiten zur Folge haben. Waren die vagi intact gelassen, und die Asynchronie der Athmung z. B. durch Trigeminusreiz hervorgerufen, so glich sich dieselbe allmälig wieder aus, und es konnten Zwerchfelle beobachtet werden, die vollkommen gleichmässig beiderseitig arbeiteten, nachdem sie sich einige Zeit vorher vollkommen versehieden und ungleichmässig contrahirt hatten, war die Asynchronie durch die beiderseitige Durchschneidung der vagi herbeigefiihrt, so dauerte der Ausgleich längere Zeit, kam aber in einigen Fällen fast vollkommen zu Stande. Die durch einen Marey'schen Hebel mit Benutzung einer Athmungsflasche während der Asynchronie aufgezeichneten Athmungseurven

1) Angeführt in den Jahresberichten über die Fortschritte der Anatomie und Physiologie. Herausgegeben von H off mann-Schwalbe 1880, Bd. VIII, 2. Abtheilung, S. 28. Selbst erschienen in Philadelphia medical Times 1879.

2) Archiv für Anatomie und Physiologie, Jahrgang 1879 und 1880.

3) Erste Mittheilung über die spinalen Centren der Athmung nach Versuchen von 0 . Langendorff und R. Nitschmann. Archiv für Anatomie and Physiologie, Jahrgang 1880, Seite 518.

4) a. a. 0 . 
zeigten eine komplicirte, zusammengesetzte Form, welche eben auf der Ungleichmässigkeit der Athmung beruhte. Letzteres konnte auf folgende Art bewiesen werden. Es wurden die Zwerchfellskontractionen beider Hälften gesondert gleichzeitig aufgezeichnet auf einem Papier ohne Ende, indem zwischen Leber und Zwerchfell jederseits kleine Gummiballons eingelegt wurden, die mit je einem Marey'schen tambour inscripteur in Verbindung standen. Zu gleicher Zeit wurde durch einen dritten Hebel die Trachealcurve aufgezeichnet, wie es oben bereits kurz angegeben ist. Setzte man nun durch Construction die beiden so gewonnenen Zwerchfellskurven zusammen, so erhielt man eine Curve, die genau ubereinstimmte mit der direct erhaltenen Trachealkurve.

In der zweiten Mittheilung ${ }^{1}$ ) über ungleichzeitige Thätigkeit beider Zwerchfellsbälften von Dr. Langendorff ist in Taf. I, Fig. 3 durch Verbindungsstriche gezeigt, wie die tiefste Inspiration in der Athmungskurve zusammenfällt mit dem gleichzeitigen Herabsteigen beider Zwerchfellhälften, und in der Curvensammlung über die eben erwähnte Arbeit befinden sich verschiedene synthetisch erbaltene und direct gezeichnete Curven, die vollkommen mit einander übereinstimmen, sie sind nicht veröffentlicht, weil sie zum Beweise weiter nicht nöthig waren. So lange das in der medulla oblongata enthaltene Athmungscentrum als das alleinige angenommen wurde, war die Erklärung der Athmungsasynchronie einfach genug. Schiff legt das Athmungscentrum in beide Seiten der medulla oblongata, Gierke weist nach, dass verbindende Faserzige $^{2}$ ) von einer Seite zur anderen gehen, durchschneidet man nun also die verbindende Brücke, so ist jedes Athmungscentrum für sich isolirt und functionirt nur für seine Hälfte. Anders wird die Erklärung, wenn nachgewiesen wird, dass das im verlängerten Marke enthaltene Centrum zwar enge mit dem Athmungsmechanismus verknïpft ist, aber nicht das Athmungscentrum direct, sondern nur ein regulatorisch wirkendes Centrum für die in der medulla spinalis enthaltenen automatisch wirkenden Centren algiebt. Ueber diesen Punkt ist eine zweite Reihe von Versuchen,

1) Archiv für Anatomie und Physiologie, Jahrgang 1881, S. 78.

2) Laura hält die sich kreuzenden Faserzüge hauptsächlich für Hypoglossusfasern, leugnet aber auch nicht einen Zusammenhang derselben mit dem Vaguskern. Hofmann-Schwalbe, Lehrbuch d. Anat. d. Menschen. II. Autl., S. 659 . 
im Anfang des Jahres 1880 von Herrn Dr. Langendorf $f^{1}$ ) und mir angestellt worden. Wäbrend Rokitansky nach Durchtrennung der medulla oblongata dicht unterhalb des calamus scriptorius nur im Strychninkramphe unvollständige Athmungen sah, und Schroff nur 2-3 Athemzïge nach derselben Operation ohne Strychninvergiftung erzielte, gelang es uns längere Athmungskurven aufzeichnen zu lassen von strychninisirten, auch nicht strychninisirten Thieren, denen das Halsmark an oben erwähnter Stelle abgetrennt war. Die besten Resultate wurden erzielt an neugeborenen oder doch nur wenige Wochen alten Thieren und zwar speciell Kätzchen, aber auch Kaninchen, die mit einer minimalen Dosis von 0,0005-0,001 Strychnini nitr. vergiftet waren. Es gelang auf diese Weise Athmungen zu erhalten, die bis 50 Minuten anhielten. Aber auch Thiere, die nicht strychninisirt waren, lieferten Athmungskurven von bedeutender Länge, wenn man längere Zeit kinstliche Athmung eingeleitet hatte und die Zeit der durch die künstliche Ventilation bedingten Apnoe voruiber liess. Bei Gelegenheit eines Vortrages des Herrn Dr. Langendorff über dieses Thema wurde der Einwand gemacht, dass auch nach Abtrennung der med. oblongata rom Rïckenmarke die nervöse Verbindung der ersteren mit den Spinalnerven keineswegs unterbrochen sei, da insbesondere der nervus phrenicus mit dem nervus hypoglossus mehrfache Anastomosen eingehe, and hierdurch eine functionelle Verbindung des bulbären Athmungscentrums mit den wichtigsten Athmungsmniskeln gegeben sein könnte. Um diesem Einwande entgegen zu treten, wurde bei einigen Versuchen nicht nur die medulla oblongata abgetrennt, sondern das Thier vollständig dekapitirt, und trotzdem blieb der Erfolg derselbe. Wollten die Thiere nicht spontan athmen, so thaten sie es auf äusseren Reiz, die strychninisirten schon auf leises Anblasen, die nicht mit Strychnin vergifteten anf Kneifen einer Pfote oder Reizung des ischiadicus oder brachialis etc.

Was die Athmung selbst anlangt, so war sie sowohl diaphragmatisch als auch costal, wenigstens bei Hunden und Katzen, denn bei Kaninchen ist sie ja immer nur diaphragmatisch. Ersteres wurde nach Eröffnung der Bauchböhle durch den Augenschein wahrgenommen, letzteres theils ebenfalls nach Freilegung der

1) Archiv für Anatomie u. Physiologie, Jahrgang 1880, S. 518. 
Thoraxmuskulatur direct geseben, theils an den Ausschlägen einer feinen Insektennadel wahrgenommen, die in die Rippenknorpel eingestochen war. Auch die Kopfathmung blieb bestehen, athmete doch nach vollständiger Dekapitation Rumpf und Kopf gesondert längere Zeit hindurch. Aus diesen Versuchen geht herror, dass in dem Spinalmarke automatisch wirksame Athmungseentren enthalten sein müssten, die auch nach Trennung von dem regulatorischen Centrum sich noch für einige Zeit eigene Functionsfähigkeit bewahren.

Wie steht es nun mit der Erklärung der durch den Longetschen Stich erzielten auf beiden Seiten ungleichmässigen Athmung. $V$ und $V$ stellen die Vaguskerne vor, $T$ und $T$ die des Trigeminus, von ihnen gehen die Faserzitge $v, v^{t}$ und $t, t^{t}$ aus, die sich zu $a$ und $a^{\prime}$ d. h. zu den beiden Respirationsbündeln vereinen. Diese beiden Respirationsbündel stehen wie Gierke mikroskopisch nachgewiesen hat in Verbindung mit einander dureh sich kreuzende Faserzüge $b$ und $b^{\prime}$. Doch ist die Kreuzung keine vollständige, sondern ein Theil der Faserzuige bleibt ungekreuzt und geht in $d$ und $d^{\prime}$ direct zum spinalen Centrum des Phrenikus $P$ und $P$, nachdem sie sich mit den gekreuzten Fasern der anderen Seite zu $c$ und $c^{t}$ vereinigt haben.

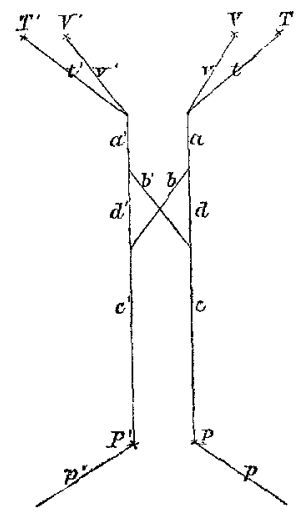
So lange nun dieses schematisch dargestellte combinirte Athmungscentrum intact ist, können Reize von allen Seiten einwirken und werden stets eine prompte gleichmässige Wirkung auf die beiderseitige Athmung ausüben. Denn trifft ein Reiz den Vagus oder Trigeminus der rechten Seite, so wird er sich durch $d$ auf das rechte Spinalcentram $P$ und durch die Gierk e'sche Kreuzung $b$ auf das linke Spinalcentrum $P^{\prime}$ fortpflanzen und beiden gleiche Impulse ertheilen. Dasselbe gilt von der linken Seite. Trifft ein Reiz den brachialis, so wird er nach einer Arbeit von Joseph ${ }^{1}$ ) nicht mebr, wie früher angenommen wurde, den Weg uber die medulla oblongata nehmen, sondern

1) Zeitmessende Versuche über Athmungsreflexe von Dr. Max Joseph. Dies Archiv Jahrgang 1883, S. 480. 
direct hinüber gehen anf die andere Seite durch das Gerlachsche Fasernetz. Ist dieser Weg aber verschlossen durch longitudinale Durchschneidung des Halsmarkes mit Bestehenlassen der Gierke'schen Kreuzung, so wird der Reiz seinen Weg uber diese Kreuzung nehmen und zur anderen Seite gelangen. Dasselbe gilt vom ischiadicus.

Nehmen wir nun an, die Brücke, welche durch die Gierkesche Kreuzung zwischen den beiden regulirenden Centren besteht, sei zerstört, so wird sich das Bild anders gestalten mïssen. Ein Reiz des vagus und trigeminus kann sich nicht mehr durch die motorischen gekreuzten Bahnen $b$ resp. $b^{\prime}$ der anderen Seite mittheilen, das entgegengesetzte Spinalcentrum erfährt nichts von dem Impulse, kann also auch nicht darauf antworten, d. h. die Wirkung auf die Athmung, die sich nur in den Bahnen $d$ resp. $d^{\prime}$ fortpflanzen kann, bleibt einseitig, und wir erhalten, da die eine Seite ruhig fortathmet, während die andere eine Störung erleidet, eine ungleichmässige Zwerchfellsthätigkeit. Hört der Reiz auf, so ist die irritirende Wirkung anfänglich doch in so weit noch anhaltend, als die Athmung für einige Zeit aus dem Gleichgewicht gebracht, sich erst allmälig beruhigt und wieder gleichmässig wird. Letzteres scheint ja auch leicht erklärlich, wenn wir bedenken, dass sobald der äussere Reiz aufhört, und die erregenden Momente fuir beide Centren wieder die gleichen werden, auch der Rhytmus sich allmälig wieder dem Athembedürfniss anpasst, und beide Hälften wieder zu regulärer Thätigkeit zurückkehren. Trifft ein Reiz irgend einen der Spinalnerven, so wird er sich auf dem gewöhnlichen Wege durch das Gerlach'sche Fasernetz der anderen Seite mittheilen, and es hat also die Durchtrennung der Brücke in der medulla oblongata auf diese Reize keinen Einfluss.

Dass eine gekreuzte Verbindung zwischen beiden Centren in der medulla oblongata bestehen muss, wie sie Gierke ja schon anatomisch nachgewiesen hat, zeigt auch ein praktischer Versuch. Wenn man das eine Respirationsbindel dicht unterhalb der Kreuzungsstelle, also dicht unterhalb der Spitze des calamus scriptorius durchtrennt, so steht die Athmung der betreffenden Seite sofort still, während die der anderen Seite fortgeht. Bestände nun keine Communication zwischen den Centren, so milssten Reize, die jetzt den vagus oder den trigeminus der verletzten Seite treffen, ohne Einfluss auf die Athmung sein, da die gereizte Körperhälfte ja 
schon an und für sich zu athmen aufgehört hat. Dem ist aber nicht so, sondern von beiden vagi aus erhält man auf die allein noch athmende eine Hälfte des Zwerchfells mit gleich starken Strömen gleiche Erfolge, die sich eben nur durch Fortpflanzing des Reizes von der verletzten Seite auf die gesunde durch directe Faserverbindung erklären lassen. Ein weiterer Beweis wiirde geliefert, wenn man das Halsmark der Länge nach spaltete, so dass eine Fortpflanzung eines Reizes, der einen brachialis z. B. trifft, durch das Gerlach'sche Fasernetz nicht möglich wird, tritt trotzdem eine Wirkung anf beide Zwerchfellshälften ein, und ist nota bene die Gierke'sche Kreuzung intact geblieben, so kann sich der Reiz nur durch diese auf die andere Seite fortpflanzen, zumal wenn es sich nachweisen lässt, dass man nur noch diese mit zu durcbtrennen nöthig hat, am sofort einen nur einseitigen Erfolg durch Brachialis-Reizung zu erzielen.

Derartige Versuche habe ich nun im Laufe des verflossenen Jahres mehrere gemacht, und sie alle haben Resultate ergeben, welche sich nicht nur mit obigen schematisch dargestellten Verhältnissen der Athmungscentren in Einklang bringen lassen, sondern erneute Beweise für die Richtigkeit derselben beibringen.

Bevor ich auf meine Versuche selbst eingehe, möchte ich noch einen Blick auf die einschlägige Literatur werfen. Die longitudinale Spaltung des ganzen Cervicalmarkes ist wohl bisher mit Rücksicht auf die Athmungsinnervation nur von Hénocque und Eloy ${ }^{1}$ ) in dem Vulpian'schen Laboratorium ausgeführt worden, wenigstens habe ich weitere Mittheilungen über diese Operation nirgends gefunden, und geben auch die beiden genannten Autoren in ihrer Arbeit keine weitere Literatur über diesen Gegenstand an. In welcher Art die Operation von Hénocque und Eloy ausgeführt ist, steht nicht angegeben, und auch über das Resultat ist nur eine kurze Mittheilung gemacht. Sie kommen $\mathrm{zu}$ folgendem Schlusse ${ }^{2}$ ): „la section longitudinale de la moelle cervicale peut être faite sans qu'il y ait arrêt de la respiration ni des contractions du diaphragme", and zwar erhalten sie diesen aus 17 Versuchen, die sie an Meerschweinchen, Katzen, Kaninchen und Hunden angestellt haben. Die Versuche an den Meerschwein-

1) Comptes rendus 1882, p. 608 .

2) Comptes rendus 1882, p. 610. 
chen 7 an Zahl sind zum grössten Theil missglüekt, indem nur zwei weiter athmeten. Bei den übrigen Thieren sind die Versuche in den meisten Fällen gelungen. Die übrigen Schlüsse sind für meine Arbeit zwar im ganzen von weniger Interesse, doch will ich dieselben anführen, da ich mir gerade über diese beiden späterhin eine Kritik erlauben muss, indem es scheinen könnte, als ob dieselben mit meinen Resultaten nicht in Einklang zu bringen wären. Sie sagen: „eine longitudinale Durehschneidung des Cervicalmarkes ausserhalb der Mitte bebt die Athmung der betreffenden Seite sofort auf", und weiter unten: „nach medianer longitudinaler Durehtrennung des Halsmarkes kann man nach einer Seite hin das Halsmark halbseitig quer durchsehneiden und erhält dabei einmal Aufhören der Athmung, das andere Mal nicht.

Dieses ist die einzige Literatur, die ich über diese Operation finden konnte, und ich komme nun zu meinen Versuchen, deren Operation ich zunächst beschreiben will.

Es wurden von mir im Ganzen 36 Versuche angestellt und zwar alle an Kaninchen. Ich wählte gerade diese Thiergattung, weil mir erstens die Thiere am leichtesten zugänglich waren, weil ausserdem die früheren Versuche über die ungleichzeitige Athmung fast ausschliesslich an Kaninchen angestellt waren, weil ferner sich die nothwendige Operation relativ leicht gerade am Kaninchenhalsmarke ausführen liess, während mich sowobl die ungünstigen Resultate, die Hénocque und Eloy mit Meerschweinchen hatten, von dieser Thierspecies abschreckten, als auch eigene Erfahrungen bei Gelegenheit frïherer Versuche mich die Untanglichkeit dieser Thiere gerade für Operationen am Halsmarke hatten erkennen lassen. Auch Brown-Séquard') theilt in einer Arbeit mit, dass die Lebensdauer bei neugeborenen Meerschweinchen nach abgetragenem Halsmarke bei weitem die kürzeste sei, nämlich $6 \mathrm{Mi}$ nuten, während dieselbe bei neugeborenen Kaninchen 34 Minuten, bei neugeborenen Hunden und Katzen sogar 48 resp. 41 Minuten beträgt. Hunde und Katzen waren nun gerade schwer zu bekommen, und da ausserdem bei der nothwendigen schwierigen und komplicirten Operation bei jeder neuen angewandten Thiergattung längere Uebung and mehrere annütze Opfer an Thieren nöthig gewesen wären, um Resultate za erzielen, so sah ich von verglei-

1) Canstatt's Jahresbericht 1853, Bd. I, S. 216. 
chenden Experimenten an verschiedenen Thieren ab und beschränkte mich lediglich auf Kaninchen. Was nun das Alter der Thiere anlangt, so babe ich allmälig gefunden, dass junge Thiere im Alter von ca. $6-10$ Wochen die geeignetsten waren. Diese jungen Thiere sind resistenter gegen Eingriffe, welche das cerebrum oder die medulla spinalis betreffen als ältere, dann sind die Wirbelbögen noch relativ leicht mit einer starken einfachen Scheere zu durchtrennen, während man bei älteren Thieren bereits Knochenzangen in Anwendung bringen müsste, die bei den engen Raumverhältnissen in denen man zu operiren gezwungen ist schwer anzulegen und zu handhaben sind. Noch jüngere Thiere andererseits, speciell neugeborene, sind auch wieder schlechter zu verwenden, da bei der Kleinheit der vorliegenden Theile, die an und für sich nicht leichte, freihändige, genau mediane Durchtrennung zur absoluten Unmöglichkeit wird. In Bezug auf das Geschlecht habe ich bei männlichen und weiblichen Thieren keinen Unterschied der Widerstandsfähigkeit gegen die Operation oder in der Blutung gefunden, ebenso wenig in Bezug auf die Farbe, nur eines fiel mir in letzter Beziehung auf und kehrte regelmässig wieder, dass speciell schwarze Kaninchen sich viel resistenter gegen Chloralbydrat erwiesen wie andersfarbige, sodass ich oft das doppelte Quantum injiciren musste, um eine kräftige Narkose herbeizuführen.

Die Operation wurde wie bereits eben angedeutet in tiefer Narkose ausgeführt, und wurde zu diesem Zwecke hanptsächlich Chloralhydrat verwandt. Es geniugte bei Thieren, welche ein Alter hatten wie es oben angegeben ist, von einer $50 \%$ Chloralhydratlösung 4-5 Theilstriche einer Pravaz'schen Spritze in die Bauchhöhle direkt zu injiciren, um nach einiger Zeit eine tiefe Narkose zu erzielen.

Bei der Operation selbst war es hauptsächlich die profuse Blutung, welche mir oft viel zu schaffen machte. Zunächst waren die Thiere, um diesem Uebelstande soviel als möglich prophylaktisch entgegen $z u$ arbeiten, längere Zeit, beziehungsweise 14 Tage, vorher auf Trockenfutter gesetzt, und dann suchte ich während der Operation durch Unterbindung, ferrum candens, Penghawar ete. derselben so gut wie möglich Herr zu werden. Versuche in einzelnen Fällen nur mit stumpfen Häkchen in den Muskelinterstitien zu operiren missglüekten, da die Blutung nicht geringer war, und 
ausserdem die irreguläre Wundfläche an Uebersichtlichkeit verlor. Die Aufspannung des Thieres erlitt insofern eine Abänderung von der gewöhnlichen Art, als dieselbe mit gekreuzten Vorderbeinen geschehen musste, um die Schulterblätter von einander zu ziehen.

Sobald die Weichtheile von den Wirbelbögen entfernt und die Blutungen gestillt waren begann der wichtige Akt der Freilegung des Markes. $\mathrm{Zu}$ diesem Zwecke fasste ich mit einer Hakenpincette durch eine vorher in die membrana oturatoria geschnittene Oeffnung den Bogen des Atlas, durchtrennte denselben zu beiden Seiten der Pincette, hob ihn heraus und schritt nun weiter fort, indem ich einen Wirbelbogen nach dem andern zu beiden Seiten der processus spinosi mit einer starken Scheere durchschnitt, vorsichtig die spitze Scheerenbranche zwischen der dura mater und dem knöchernen Kanal vorschiebend. Sobald einige (3-4) Wirbelbögen durchtrennt waren, nusste der Assistent mit den beiden Pincetten die Weichtheile von neuem etwas mehr unterhalb fassen, damit der zu durehtrennende Wirbelbogen stets der am höchsten ${ }^{e}$ und am bequemsten gelegene sei. Auf diese Weise gelang es mir stets die sämmtlichen processus spinosi zusammenhängend zu entfernen und eine Rinne in den Cervicalkanal zu schneiden, durch welche das Halsmark in seiner ganzen Ausdehnung zu Tage trat.

Sobald die Blutung vollkommen stand, was durchaus nothwendig ist, da man zu den folgenden Operationen das Operationsfeld gut übersehen muss, wurde mit einer feinen Hakenpincette die in der Rautengrube bereits verletzte dura mater gefasst, angehoben und ein feines, scharfes Messer mit der Schneide nach oben zwischen medulla und dura mater geschoben und der ganze Duralsack der Länge nach gespalten.

$\mathrm{Za}$ der nun folgenden Durchtrennung des Markes in der Medianlinie benutzte ich anfänglich eine breite Staarnadel, später aber liess ich mir ein eigenes Messerchen dazu anfertigen, das die Form eines feinen Stemmeisens hatte. Die schneidende Fläche selbst war unten $2 \mathrm{~mm}$ breit, hatte eine Höhe von $4-5 \mathrm{~mm}$ und lief dann nach oben in einen soliden Stiel aus. Sämmtliche drei iibrigen Seiten dieses kleinen genau rechtwinkligen Stemmeisens waren scharf geschliffen, und es musste daher das Instrument von bestem Material gearbeitet sein, da es, um obige Eigenschaften zu haben, nur...sehr fein und dünn gearbeitet sein durfte und doch 
eine gewisse Haltbarkeit besitzen musste, um bei eventuellen Zuckungen des Thieres nicht sofort abzubrechen. Dieses Messerchen wurde genau senkrecht und genau in der Medianlinie aufgesetzt, in die medulla spinalis hineingesenkt und dann mit kurzen, langsamen, sägeförmig stossenden Zügen allmälig die ganze medulla durchtrennt. In der Medianlinie verläuft die arteria medullae spinalis posterior, und da diese beim Anschneiden etwas blutet und dadurch das Operationsfeld verdunkelt, fing ich die Durchtrennung stets von oben an, da die Arterie einmal durchtrennt unterhalb nicht mehr bluten kann, während ich sonst, wenn ich die Arterie von unten auf durchtrennt hätte, immer gerade an der blutenden Stelle hätte operiren müssen. Die sägeförmigen Zïge waren deshalb nothwendig; da bei dem Versuche einen glatten ziehenden Schnitt $z u$ machen, das Halsmark sich etwas vor dem Messer herschob, aus seiner normalen Lage kam und man nun nicht mehr mit Sicherheit in der Medianlinie bleiben konnte.

War die Operation glücklich vollendet, so wurde die ganze Wundfläche mit Penghawar bedeckt und die Haut darüber mit Klemmpincetten geschlossen. Dann wurde das Thier los gebunden auf den Rücken gelegt, sofort die Bauchhöhle eröffnet und die Contractionen des Zwerchfells direct beobachtet. Hatte das Thier aufgehört zu athmen und war künstliche Athmung nöthig, so wurde schnell Tracheotomie gemacht, und während der Zeit durch eine iiber die Schnauze gestülpte Gummikappe ventilirt. Die weiteren Operationen, die noch zu den Versuchen nöthig waren, waren die Freilegung der ischiadici oder brachiales oder vagi. Es wurden die betreffenden Nerven, die zu dem Versuche gebraucht werden sollen, auf beiden Seiten gleichmässig freigelegt, in HartgummiPlatinelektroden gebettet und durch eine electrodenwechselnde Wippe mit dem Inductionsapparate in Verbindung gebracht, die eine abwechselnde Reizung beider Nerven erlaubte. Auf diese Weise war es leicht, einen gleichen Reiz durch einen Druck von einem Nerven auf den anderen wirken zu lassen und den Unterschied der Wirkung zu sehen.

Von jedem gelungenen und registrirten Versuche wurde das Halsmark in Mïll er'scher Flüssigkeit,oder Alkohol gehärtet und später der Schnitt auf seine Ausdehnung, Vollständigkeit und Richtung mit der Lupe untersucht.

Die Versuche selbst, die ich auf oben angegebene Art anstellte, 
zerfallen in drei Gruppen. Bei der ersten Gruppe durehtrennte ich das Halsmark nur bis dicht unterhalb der Spitze des calamus scriptorius, bei der zweiten durchtrennte ich die Spitze selbst auch mit und bei der dritten sollte das Halsmark durchtrennt werden nach vorangegangener Abtragung der medulla oblongata. Bevor ich in die Versuchsreihe der Gruppe I eintrat, wiederholte ich noch einige der La ngendorff'schen Versuche, die ich hier nicht mehr anführen will, da sie dasselbe Resultat lieferten, das oben bereits angegeben ist.

\section{Versuchsreihe 1.}

Versuch VIII. Kleines, graues, 8 Wochen altes, männliches Kaninchen. Es bekommt zunächst 4 Theilstriche Chloralhydrat, worauf das Halsmark freigelegt wird. Beim Versuch, die medulla spinalis zu spalten, zuckt das Thier und bekommt daher noch weitere 11/2 Theilstriche. Die mediane Spaltung wird nun ausgeführt und zwar vom ersten bis siebenten Halswirbel Die Athmung geht anscheinend ruhig fort, als jedoch das Abdomen eröffnet wird, zeigt es sich, dass nur noch die rechte Zwerchfellshälfte functionirt, während die linke still steht. Zur Untersuchung des Einflusses der vagi auf die Athmung werden diese freigelegt und nach einander durcbschnitten und zwar zunächst der rechte. Die Athmung steht momentan still, setzt aber bald wieder ein und geht in verlangsamtem Tempo allein auf der rechten Seite weiter. Darauf Durchschneidung des linken vagus, die Athmung steht wiederum still und setzt erst ein, nachdem eine kurze Zeit künstliche Athmung eingeleitet ist. Hierauf sollte das Halsmark in der Höhe des zweiten Brustwirbels abgetragen werden, worauf das Thier an Verblutung zu Grunde geht.

Die spätere Untersuchung des gehärteten Halsmarkes hat ergeben, dass der Schnitt vom ersten bis siebenten Halswirbel reicht und ca. $2 \mathrm{~mm}$ unter der Spitze des calamus scriptorius beginnt. In der Höhe des zweiten und dritten Halswirbels ist derselbe an der vorderen Fläche etwa $1 \mathrm{~mm}$ von der Medianlinie abgewichen und in die Marksubstanz der linken Seite hineingedrungen. Auf der hinteren Fläche verläuft er genau in der Medianlinie.

Versuch X. Kleines, graues, 8 Wochen altes, weibliches Kaninchen. Es bekommt zunächst 3 Theilstriche Chloralhydrat, nach der Freilegung der medulla spinalis noch weitere 2 Theilstriche. Die Spaltung gelingt vom ersten Halswirbel bis zum ersten Brustwirbel. Die Athmung geht nur auf der linken Seite fort. Die beiden vagi werden wieder freigelegt und nach einander durchschnitten. Wieder ist der Erfolg derselbe, von beiden Seiten aus treten kurze Athmungspausen ein, denen eine verlangsamte und vertiefte Athmung folgt. Nun werden die trigemini gereizt, durch Aufpioseln von Chloroform auf die Nasenschleimhaut, von beiden Seiten aus tritt Athmungs- 
stillstand ein. Als das Rückenmark wieder in der Höhe des zweiten Brustwirbels durchtrennt werden soll, tritt wieder der Verblutungstod ein.

Die Untersuchung des gehärteten Halsmarkes mit der Lupe lässt keine Abweichung des Schnittes erkennen, sondern derselbe verläuft sowohl vorne wie hinten vom ersten Halswirbel bis zum ersten Brustwirbel genau in der Medianlinie. Allexdings hatte das Thier bei Gelegenheit der medianen Spaltung in der Höhe des dritten bis vierten Halswirbels gezuckt, und mag dabei die rechte Hälfte des Cervicalmarkes etwas gequetscht worden sein.

Versuch XII. Kleines, schwarzes, 8 Wochen altes, männliches Kaninchen. Es bekommt allmälig 10 Theilstriche Chloralhydrat bevor mit der Operation überhaupt begonnen werden kann und dann nach Freilegung des Halsmarkes noch weitere 2 Theilstriche. Die Durchtrennung gelingt vom zweiten bis zum siebenten Halswirbel, darauf ist die Athmung anfänglich sehr schwach und oberfächlich aber beiderseitig. Es wird längere Zeit $(1 / 4$ Stunde) künstlich geathmet, und nun wird die Athmung beiderseitig tief diaphragmatisch. Reize, die von dẹn vagi und den trigemini ausgeübt werden, haben wiederum gleiche Erfolge auf beide Zwerchfellshälften. Es werden nun die brachiales frei gelegt und mit Hartgummi-Platinelectroden behufs electrischer Reizung, wie oben bereits angegeben wurde, versehen, und auch von diesen aus erhält man nach einseitigen Reizen eines der beiden brachiales, von beiden Seiten auf beiden Seiten gleichzeitig A thmungsstillstand oder auch nur gleichmässige Verlangsamung je nach der Intensität des Reizes.

Die Untersuchung zeigt den Schnitt in der Ausdehnung vom zweiten. bis siebenten Halswirbel vorne sowohl wie hinten genau median verlaufend.

Was die Menge des angewandten Chloralhydrats anbetrifft, so habe ich oben bereits gesagt, dass es mir bei allen schwarzen Thieren auffiel, dass sie mehr Chloralhydrat verlangten. Dieses Thier speciell zeigte sich ganz besonders resistent gegen die Chloralnarkose, und kann ich mir diese Erscheinung nur so erklären, dass die ersten vier Theilstriche, die in die Bauchhöhle injicirt wurden, vielleicht aus Versehen, ich habe die erste Injection in diesem Falle nicht selbst gemacht, in den Darm gelangt sind und so ohne Wirkung blieben, von den anderen Dosen weiss ich sicher, dass sie nicht in den Darm gelangt sind, und es bleiben da immerhin noch acht Theilstriche übrig, die zur Narkose nöthig waren.

Versuch XV. Kleines, graues, 7 Wochen altes, männliches Kaninchen bekommt zunächst 3 , später noch $11 / 2$ Theilstriche Chloralhydrat. Die Durchschneidung wird ausgeführt vom ersten Halswirbel bis zum ersten Brustwirbel. Die Athmung geht nur auf der rechten Seite gut fort, während es auf der linken Seite hin und wieder den Anschein hat, als mache sie kleine mit der rechten Seite synchronische Athmungen, doch wird diese Hälfte jedenfalls nur von der rechten Seite, wenn die Contractionen derselben zufällig etwas stärker waren, mitgezogen sein. Die Vagi werden frei präparirt und electrisch gereizt. Bei einem Daniell'schen Element und einem Rollenabstand des Dubois'- 
schen Schlittenapparates von $25 \mathrm{~cm}$, wird die Athmung der rechten Seite von beiden Vagi aus gleichmässig verlangsamt, bei $18 \mathrm{~cm}$ ebenfalls von beiden Seiten aus zum Stillstande gebracht. Die Reizung des Trigeminus ergiebt wieder dieselben Resultate wie früher.

Die Durchtrennung hat stattgebabt vom ersten Halswirbel bis zum ersten Brustwirbel und zwar verläuft der Schnitt hinten genau median, vorne ist er in der Höhe zwischen dem ersten und zweiten Halswirbel etwas nach links abgewichen, kehrt aber sofort in die Medianlinie zurück und bleibt genau in derselben tois zum Schlusse.

Versuch XVII. 10 Wochen altes, graues, weibliches Kaninchen bekommt zunächst 5, später noch $1 \frac{1}{2}$ Theilstriche Chloralhydrat. Die Durchschneidung wird ausgeführt vom ersten Halswirbel bis zum ersten Brustwirbel. Die Athmung geht beiderseitig fort. Nun wird der linke Vagus unterbunden, worauf die Athmung 5 Minaten lang steht, dann verlangsamt weiter geht sich allmälig wiederholend. Nachdem sie zu alter Frequenz zurückgekehrt ist, wird der rechte Vagus unterbunden, worauf sich dieselbe Erscheinung nur prägnanter wiederholt. Bei electrischer Reizung der Vagi erbält man bei $18 \mathrm{~cm}$ Rollenabstand von beiden Seiten aus auf beiden Seiten Stillstand der Respiration, bei $20 \mathrm{~cm}$ nur Verlangsamung ebenfalls mit gleichem Erfolg von beiden Seiten auf beiden Seiten. Die electrische Reizung der Brachiales liefert auch bei $15 \mathrm{~cm}$. Rollenabstand von einer Seite aus auf beiden Seiten Athmungsstillstand, desgleichen die Reizung der Ischiadici. Es soll nachträglich noch die Spitze des Calamus scriptorius durchtrennt werden, dabei hört jedoch das Thier zu athmen auf und ist auch nicht mehr durch künstliche Athmung zu erneuter Respiration zu bringen.

Die Untersuchung des Schnittes zeigt ihn vorne wie hinten genau median vom ersten Halswirbel bis zum ersten Brustwirbel verlaufend.

Versuch XVIII. Kleines, schwarzes, fünf Wochen altes, weibliches Kaninchen bekommt zunächst 6, später noch 2 Theilstriche Chloralhydrat. Nach der Durchschneidung der Medulla spinalis vom ersten bis siebenten Halswirbel athmet das Thier nur auf der linken Seite. Reize des linken Brachialis haben dieselbe Wirkung auf die Athmung, wie die des rechten. Es wird die Medulla oblongata in der Höhe der Spitze des Calamus scriptorius quer durchtrennt, worauf die Athmung still steht.

Das Mark ist vom ersten bis siebenten Halswirbel vorne sowohl wie hinten fast genau median durchtrennt, nur in der Höhe des dritten Halswirbels weicht der Schnitt vorne um ein Minimum (kaum $1 / 2 \mathrm{~mm}$ ) von der Medianlinie ab, in die er sofort wieder zurïckkehrt. Die quere Durchtrennung hat in der Höhe der Spitze des Calamus scriptorius stattgehabt.

Das Resultat dieser Versuchsreihe ist also in kurzen Worten folgendes: Es lässt sich das in dem Halsmarke enthaltene Athmungscentrum durch einen Längsschnitt in zwei Hälften theilen, 
die beide rubig weiter thätig bleiben, wenn der Schnitt sich genau in der Medianlinie gehalten hat, doch genügt eine geringfügige Abweichung nach einer der beiden Seiten, um die Thätigkeit der betreffenden Zwerchfellhälfte sofort aufzuheben.

Wenn beide Hälften athmen, so thun sie dieses stets regelmässig und synchron und jeder Reiz der vom vagus oder trigeminus oder brachialis oder ischiadicus ausgeht, übt gleiche Wirkung auf beide athmende Hälften. Es besteht also in der medulla oblongata ein regulirendes Centrum, von welchem ans die in dem Halsmarke enthaltenen Centren kontrollirt werden.

In der zweiten Versuchsreihe wurde nun also dieses regulirende Centrum auch noch gespalten.

\section{Versuchsreihe II.}

Versuch XIV. Dieser Versuch wurde, wie ja auch die Nummer zeigt, eigentlich innerbalb der Versuchsreihe I gemacht, ergab aber ganz andere Resultate, die so frappant den bis dahin gefundenen geradezu widersprachen, dass ich sie mir anfänglich nicht erklären konnte. Die spätere Untersuchung des gehärteten Markes hat nun aber ergeben, dass zufällig der Schnitt bereits in der Spitze des Calamus scriptorius begann anstatt dicht unterhalb derselben, sodass der Versuch also in die Versuchsreihe II gehört, wohin er mit seinen Resultaten allerdings auch genau hinpasst.

9 Wochen altes, graues, weibliches Kaninchen; es bekommt anfânglich 4, später noch $1 \frac{1}{2}$ Theilstriche Chloralhydrat. Die Durehtrennung erfolgt vom Calamus scriptorius bis zum ersten Brustwirbel. Die Athmung persistirt nur auf der linken Seite. Nach Freilegung der Vagi verursacht ein electrischer Reiz bei $18 \mathrm{~cm}$ Rollenabstand von der linken, also der athmenden Seite aus Athmungsstillstand, während von der rechten, der nicht athmenden Seite aus auch bei völlig übereinander gesehobenen Rollen kein Einfluss auf die Athmung ausgeïbt wird, sondern dieselbe geht rubig rhytmisch auf der linken Seite fort. Dasselbe Resultat erhält man durch Reizung des Trigeminus. Es werden die Plexus brachiales freigelegt und auch von diesen übt nur der linke, d. h. der der gesunden Seite einen Einfluss aus, (bei $12 \mathrm{~cm}$ Rollenabstand Stillstand der Athmung), während auch die stärksten Ströme von der rechten Seite die Athmung nicht beeinträchtigen. Schliesslich werden auch noch die Ischiadici freigelegt und von diesen erhält man von beiden Seiten aus bei $10 \mathrm{~cm}$ Rollenabstand Athmungsstillstand.

Die Untersuchung des gehärteten Markes hat folgendes Ergebniss. Der Schnitt verläuft von der Spitze des Calamus scriptorius bis zur Höhe des zweiten Brustwirbels hinten genau in der Medianlinie, vorne biegt er in der Höhe des zweiten Halswirbels nach rechts aus und verläuft von da parallel mit der Medianlinie in einer Entfernung von $1 \mathrm{~mm}$ bis zum Sehlusse. 
Versuch XXIV. Kleines, graues, 4 Wochen altes, männliches Kaninchen. Es bekommt 3, später noch $1 \frac{1}{2}$ Theilstriche Chloralhydrat. Die Durchtrennung wird gemacht von dem unteren Winkel der Rautengrube an bis zum letzten Halswirbel. Die Athmung besteht nur auf der rechten Seite. Aaf Reiz des rechten Vagus bei $25 \mathrm{~cm}$ Rollenabstand Stillstand der Athmung, vom linken Vagus auch bei $10 \mathrm{~cm}$ noch kein Einfluss. Weiter wurde der Versuch nicbt gemacht.

Der Schnitt geht von dem unteren Winkel der Rautengrube bis zum ersten Brustwirbel, hält sich hinten genau in der Medianlinie, vorne weicht er nur in der Höhe des Calamus soriptorius um $1 / 2 \mathrm{~mm}$ nach links ab, lenkt dann wieder in die Medianlinie ein und verläuft in derselben bis zu seinem Ende.

Versuch XXVI. 8 Wochen altes, schwarzes, männliches Kaninchen bekommt anfänglich 6, später noch 2 Theilstriche Chloralhydrat. Da das Thier wiederbolentlich bei der Durchtrennung des Markes zuckt, muss dieselbe öfter unterbrochen und immer wieder von Neuem begonnen werden. Sie gelingt schliesslich von dem unteren Winkel der Rautengrube bis zum 7ten Halswirbel. Das Thier athmet nach der Operation beiderseitig. Die Electroden werden an die vagi angelegt und man bekommt sofort ungleichmässige Athmung schon herbeigeführt durch das Anlegen der Elektroden allein.

Bei Reizung des linken vagus bei $18 \mathrm{~cm}$ Rollenabstand Aufhören der Athmung nur auf der linken Seite, während die rechte ruhig weiter athmet; ebenso bei Reizung des rechten vagus nur Stillstand der rechten Zwerchfellhälfte, während die linke ruhig weiter athmet. Leider kam das Thier darauf sehr bald zum exitus, sodass weitere Versuche nicht angestellt werden konnten.

Der Schnitt lief von der Spitze des calamus scriptorius bis zum 7 ten Halswirbel und zwar bis zum 4ten Halswirbel sowohl vorne wie hinten vollkommen median, von da ab biegt er vorne nach links ab und vom 6ten Halswirbel auch hinten, sodass in der Höhe des 6ten und 7 ten Halswirbels der Schnitt nur noch in der Substanz der linken Markhälfte verläuft.

Versuch XXVIII. 8 Wochen altes, graues, weibliches Kaninchen bekommt 4, später noch $1 \frac{1}{2}$ Theilstriche Chloralhydrat. Der Schnitt wird geführt von der unteren Spitze der Rautengrube bis zum letzten Halswirbel. Die Athmung persistirt nur links. Fom linken vagus aus bei $20 \mathrm{~cm}$ Rollenabstand Stillstand der Athmung, vom rechten kein Erfolg. Darauf werden die Electroden an die brachiales angelegt, und auch hier lässt sich ein Einfluss auf die Athmung nur von der gesunden Seite aus erzielen, indem sie bei $16 \mathrm{~cm}$ Rollenabstand steht, während von dem rechten plexus brachialis aus kein Einfluss ausgeibl wird.

Der Schnitt verläuft hinten genau median in einer Ausdehnung vom calamus scriptorius bis zum 7 ten Halswirbel, vorne weicht er in dèr Höhe des 2ten Halswirbels um $1 / 2 \mathrm{~mm}$ nach rechts ab, kehrt aber gleich wieder in die Medianlinie zurück, um darin bis zum Schluss zu verlaufen. 
Versuch XXX. Kleines, graues, 6 Wochen altes, weibliches Kaninchen bekommt 4, später noch 2 Theilstriche Cloralhydrat. Der Schnitt wird gemacht von der Spitze der Rautengrube bis zum 6ten Halswirbel. Die Athmung steht links und geht nur rechts weiter. Vom rechten vagus bekomme ich bei $20 \mathrm{~cm}$ Rollenabstand Stillstand, während ich vom linken auch bei $10 \mathrm{~cm}$ noch keinen Erfolg habe. Von den ischiadici erhalte ich bei $15 \mathrm{~cm}$ von beiden Seiten aus Stillstand.

Der Schnitt läuft vom calamus scriptorius bis zum 7 ten Halswirbel linten genau median, vorne jedoch ist er bereits in der Höhe des 1ten Halswirbels um $1 \mathrm{~mm}$ nach links abgewichen und verläuft in dieser Entfernung seiner ganzen Länge nach parallel mit der Medianlinie.

Versuch XXXI. 7 Wochen altes, graues weibliches Kaninchen, erhält 4, später noch $1 \frac{1}{2}$ Theilstriche Chloralhydrat. Die Durchtreunung des Halsmarkes gelingt von der Spitze der Rautengrube an bis zum 7 ten Halswirbel. Nach der Operation athmet das Thier beiderseitig. Auf Reize der vagi, auch der trigemini kann man Verlangsamung oder auch Stillstand der betreffenden einen Zwerchfellhälfte erzielen. Es wird nun die medulla spinalis von der medulla oblongata dicht unterhalb der Spitze des calamus scriptorius abgetrennt, darauf steht die Athmung still und es ist auch durch Strychnin weder spontan noch reflectorisch eine Athmung zu erzielen. Der Schnitt verläuft in seiner ganzen Ausdehnung vom calamus scriptoris bis zum 7 ten Halswirbel genau median sowohl vorne wie hinten, und die Abtragung des Halsmarkes von der medulla oblongata ist durch einen glatten Schnitt dicht unterbalb der Spitze des calamus scriptorius gelungen.

Das Resultat dieser zweiten Reihe von Versuchen ist demnach folgendes: Wenn man mit dem Halsmarke zugleich auch die Spitze des calamus scriptorius durchtrennt, so baben Reize, die die vagi, trigemini oder brachiales treffen, nur einseitige Wirkung und zwar anf derselben Seite des ausgeübten Reizes, während Reize, die rom ischiadicus ausgehen, auch in diesem Falle noch auf beide Seiten wirken.

Wie ich in der Disposition meiner Versuche angab, war es mein Vorhaben diesen beiden Versuchsreihen noch eine dritte an zu fügen, welche die longitudinale Durchtrennung des Halsmarkes nach voraufgegangener Abtragung der medulla oblongata dicht unterhalb der Spitze des calamus scriptorius bezwecken sollte. Ich babe auch einige Versuche in diesem Sinne angestellt, ohne jedoch dabei irgend etwas erreicht zu haben, indem die Thiere den zweiten Eingriff im Halsmarke nach der bereits voraufgegangenen Ruckenmarksdurchtrennung nicht mehr vertrugen, sondern sofort $\mathrm{zu}$ athmen aufhörten, und keine Athmung weiter weder durch Strychnin- 
vergiftung noch durch längere Zeit fortgesetzte künstliche Respiration $z u$ erhalten war. Nach einigen in diesem Sinne unnütz geopferten Thieren, nahm ich daher von weiteren Versuchen Abstand, zumal ich auch von einem gelungenen Versuche keine Beweise weiter erwarten durfte, die icb nicht bereits durch die angeführten Versuche erbalten bätte.

Was ich nun durch diese Arbeit beweisen wollte und auch glaube bewiesen $\mathrm{zu}$ haben ist folgendes:

Ausgebend von der Voraussetzung, dass im Cervicalmarke automatisch wirksame Athmungscentren enthalten seien, wie es durch die am Anfange dieser Arbeit angeführte Literatur bewiesen ist, wollte ich untersuchen ob diese Centren, ähnlich wie das in der medulla oblongata enthaltene, theilbar seien in der Art, dass sie gesondert für je eine Körperbälfte functioniren könnten, zumal Schiff ${ }^{1}$ ) gefunden hat, dass die Fortleitung der Empfindung in jeder Rückenmarkshälfte gesondert möglich ist. Das Resultat stellt sich nun so:

I. Es lassen sich die cervicalen Centren durch einen longitudinalen Schnitt von einander trennen, ohne dass die Athmung irgend eine Veränderung erfährt, so lange die Durchtrenung genau in der Mittellinie geschehen ist. Schon eine minimale Abweichung von letzterer nach einer der beiden Seiten hat den Stillstand der Athmung auf der betreffenden Seite zur Folge. Jedoch erst vom 4. Halswirbel an aufwärts. Bei meinen ersten Versuchen, bei denen ich die Durchtrennung des Markes von unten begann nach oben aufsteigend, habe ich vielfach die Erfahrung gemacht, dass selbst gröbere Abweichungen nach der Seite, unterhalb des 4 . Halswirbels auf die Athmung nicht störend einwirkten, das Thier machte regelmässig energisehe, zuckende Bewegungen, sobald sich diese aber beruhigt hatten, ging die Athmung wieder beiderseitig normal fort, wenn aber die Abweichung von der Mittellinie vom 4. Halswirbel an aufwärts stattgefunden hatte, traten zunächst auch dieseiben Zuckungen ein, bei ihrem Aufhören jedoch stand regelmässig die eine Seite der Athmung still, und wie die spätere Untersuchung erwies regelmässig die Seite, auf welcher die Verletzung stattgehabt hatte. Dieses Faktum ist auch aus den angeführten Ver-

1) Lehrbuch der Physiologie des Menschen. Bahr 1858-1859, S. 264, 
suchen leicht ersichtlich, bei jedem verzeichneten Athmungsstillstand hat die später erfolgte Untersuchung eine Verletzung auf der betreffenden Seite zwischen der medulla oblongata und dem 4. Halswirbel ergeben, während Verletzungen unterhalb dieser Stelle von keinem Einflusse waren, wie theils die Erfahrungen bei der Operation selbst lehrten, wie oben bereits gesagt ist, theils der eine Versuch in sehr schöner Weise erkennen liess, bei welchem die Athmung beiderseitig bestanden hatte, obwohl der Schnitt vom 4. Halswirbel an nach links gewichen war, sodass er schliesslich vom 6 . Halswirbel an gauz in der linken Markhälfte verlief. (Versuch 26.)

Es konnte also die Athmung nach longitudinaler Spaltung der spinalen Centren bei exacter Operation intact erhalten werden, sie konnte dann aber auch nicht durch einseitige Reize unregelmässig gemacht werden, so lange noch eine Communication des regulatorischen Centrums in der medulla oblongata durch die Gierke'sche Kreuzung von einer Seite zur anderen bestand. Erst sobald diese mit durchtrennt war, wurde die Athmung auf beiden Seiten ungleichmässig, und ich fand also:

II. Nach longitudinaler Durchtrennung des Halsmarkes und des calamus scriptorius bleibt die Athmung beiderseitig gleichmässig bestehen, wird aber auf Reize, die die vagi, trigemini und brachialis treffen ungleichmässig, während Reize der ischiadici auf beide $Z$ werchfellshälften gleichmässig wirken.

Lag schon ein Beweis für die Theilbarkeit des spinalen Atbmungscentrums in dem Umstande, dass es möglich war die eine Hälfte der Zwerehfellsathmung durch geringe Verletzung einer Seite des Halsmarkes ausser Function zu setzen, während die andere thätig blieb, so ist in der zweiten Versuchsreihe noch ein erneuter Beweis dadurch geliefert, dass es gelungen ist beide Hälften thätig zu erhalten und ungleichmässig wirken $\mathrm{zu}$ lassen.

Wurde nur die Gierke'sche Kreuzung durchstochen, so gelang es von den brachiales aus nicht eine ungleichmässige Wirkung auf beide Zwerchfellhălften zu erzielen, da die spinalen Centren eben direct durch sensible Bahnen in Verbindung stehen. Waren diese sensiblen Bahnen allein durchtrennt, so war dieses anch nicht genügend, um einseitige Wirkung zu erhalten, da sich der Reiz auf das Centrum in der medulla oblongata fortpflanzte und von hier auf den motorischen Bahnen durch die Gierke'- 
sche Krenzung beiden Seiten der spinalen Centren zugefuhrt wurde. Wurde nun aber diese Brücke auch zerstört, so erhielt man von den brachialis aus einseitige Wirkung $d$. h. die beiden Hälften der spinalen Centren waren von einander getrennt und functionirten auf einseitigen Reiz auch nur einseitig, während sie auf Reize, die beide gleichmässig trafen z. B. von den ischiadici ausgehend auch gleichmässig reagirten. Es theilt sich eben der Reiz, der einen ischiadicus trifft, durch die quer verlaufenden, sensiblen Fasern im Rückenmarke anch der anderen Hälfte des Rïckenmarkes noch unterhalb der Durchschneidungsstelle mit und ïbt gleichmässig auf beide spinalen Centren einen Impuls aus, der zur gleichmässigen Thätigkeit beider führt.

Es lässt sich also nicht nur das in der medulla oblongata enthaltene Centrum in zwei Hälften zerlegen, sondern auch die spinalen Centren im Cervicalmarke.

In wiefern lassen sich meine Resultate nun mit denen von Hénocque $e^{1}$ and Eloy gefundenen vereinbaren.

Ihren ersten Satz, dass man das Halsmark in seiner ganzen Länge spalten kann, ohne dass die Athmung nothwendiger Weise aufhöre, habe ich vollkommen bestätigen können. Ihren zweiten Satz, dass die Athmung einer Seite steht, sobald man die betreffende Halsmarkshälfte der Länge nach spaltet, habe ich ebenfalls bestätigen können, ausserdem aber noch gefunden, dass bereits eine geringe Verletzung einer Halsmarkshälfte dicht neben der Medianlinie genügt, um die Athmung der betreffenden Zwerchfellshälfte sofort aufzuheben. Der dritte Satz jedoch, den die beiden genannten Autoren aufstellen, stimmt mit meinen Resultaten durchaus nicht überein. Sie sagen, wenn man ein Halsmark der Länge nach gespaltet hat, so kann man diesem longitudinalen Schnitt nach einer Seite quere Durchschneidungen einer Hälfte des Markes hinzufïgen und erhält dabei verschiedene Resultate.

Schon der Umstand, dass die genannten Autoren selbst angeben, verschiedene Resultate erhalten $\mathrm{zu}$ haben, spricht nicht für die Zuverlässigkeit ihrer Beobachtungen, einmal soll nach einem solchen Schnitt zwischen dem dritten und vierten Halswirbel bei einer Katze die Atbmung völlig d. h. beiderseitig aufgehört haben, das andere Mal bört die Athmung nach einer halbseitigen Durch-

1) a. a. 0 . 
schneidung über der vierten Spinalwurzel also am unteren Ende des vierten Halswirbels für 8 Minuten auf und kehrt dann wieder, und das dritte Mal wird die eine Halsmarkhälfte in verschiedenen Höhen im dritten, vierten, sechsten und siebenten Intervertebralgelenke durchtrennt und erst nach dem letzten Schnitt hört die Athmung auf. $O b$ die genannten Autoren bei diesen Versuchen sich jedesmal nach ausgeführtem Längsschnitte von der doppelseitigen Athmung überzeugt haben, steht nicht angegeben, und ich glaube es nicht, da ich einmal ihre Resultate sonst mit den meinigen nicht vereinen könnte, und dann schliesse ich es aus einer Bemerkung an einer anderen Stelle, wo über andere Versuche gesprochen wird und zwar über die Ausreissung der Wurzeln der nervi phrenici. Sie sagen an der Stelle ${ }^{1}$ ): „Wenn man die Wurzeln des phrenicus einer Seite ausreisst, so wird die Athmung unruhig, kurz, stossend und rundet sich erst allmälig ab, öffnet man nun das Abdomen, so sieht man, dass die eine Zwerchfellshälfte sich nicht mehr kontrahirt", ob diese aber ihre Thätigkeit sofort nach der Operation eingestellt hat, und ob die unruhigen Athmungen nur von der einen $Z$ werchfellshälfte ausgefuhhrt sind, bleibt unentschieden. Daher glaube ich, dass auch in diesen Fällen die Beobachtung des Zwerchfelles von vorne herein unterblieben ist, andererseits aber kann man von aussen kaum erkennen, ob eine oder beide Zwerchfellshälften athmen. Ausserdem habe ich gerade in den Fällen, in denen es mir darauf ankam, genau den Verlauf des Schnittes zu wissen, eine Angabe darüber, die auf eine spätere, exacte Untersuchung hätte schliessen lassen, vermisst, so dass ich in einigen Fällen in Ungewissheit geblieben bin, ob die Schnitte auch in Wirklichkeit den Verlauf gehabt haben, den sie hätten haben sollen. Ich nehme daher an, dass im ersteren Falle entweder nur die linke Hälfte noch in Thätigkeit gewesen ist, und nach der Durchtrennung dieser Seite des Halsmarkes auch aufgehört hat zu athmen, oder dass beim Eingehen mit dem Messer zur queren Durchtrennung auch die rechte Seite dicht neben der Medianlinie etwas verletzt ist, was nach meinen Versuchen zur Aufhebung der Athmung genügt, und so auch diese Hälfte ausser Function gesetzt ist, auch wenn sie vorher geathmet hat. Der zweite Versuch stimmt mit meinen Erfahrungen überein, denn unterhalb des vierten Halswirbels babe auch ich die Seiten des

1) Comptes rendus. Jahrgang 1882, S. 574.

E. Pfinger, Archiv f. Physiologie. Bd. XXXV. 
Halsmarkes verletzen können ohne Athmungsstillstand zu erhalten. Die für kurze Zeit eingetretene Apnoe findet ihre Erklärung in dem mit dem Eingriff verbundenen Reiz.

Den dritten Fall kann ich mir wieder nur dadurch erklären, dass die betreffende Seite entweder überhaupt nicht mehr geathmet hat und Zerstörungen ihrer Halsmarkshälfte in Folge dessen keinen Einfluss mehr hatte, oder wenn sie geathmet hat, so ist sie allein zum Stillstande gekommen, und die andere Hälfte kontrahirte sich ruhig fort und täuschte vollkommene Athmungen vor. Dass die Athmung bei Durehtrennung in der Höhe des siebenten Intervertebralgelenkes plötzlich stand, findet eine leichte Erklärung in einem zufälligen Reiz, den die allein noch athmende Seite bei Gelegenheit der Durchtrennung der anderen Seite traf und genïgte das an und für sich durch die Operation bereits geschwächte Centrum ganz zum Verlöschen zu bringen. Wer Operationen am Halsmarke zu Athmungsversuchen gemacht hat, wird es erfahren haben, wie oft die minimalsten Schädlichkeiten genügen, um die bereits geschwächten Athmungscentren abzutödten, und wie andererseits die Eröffnung der Bauchböhle zur directen Untersuchung der Zwerchfellsthätigkeit durchaus nothwendig ist, da man durch den äusseren Augenschein zu leicht getäuscht werden kann.

Durch den Nachweis, dass im Spinalmarke automatisch wirksame Athmungscentren liegen, die ebenso wie das in der medulla oblongata enthaltene theilbar sind und ihre gesonderten Functionen für je eine Seite des Zwerchfells haben, dürfte zur Genüge erwiesen sein, dass ein alleiniges Athmungscentrum in der medulla oblongata nicht vorhanden ist, und ohne dem verlängerten Marke den wichtigen Einfluss absprechen zu wollen, den es auf die Athmung hat, müssen wir ihm doch spinale Centren zur Seite stellen, die nicht minder wichtig sind.

Wer freilich ein einheitliches Coordinationscentrum für den gesammten Athmungsmechanismus verlangt, der hat dasselbe in der augenblicklichen Lösung der Frage auch nicht gefunden, doch liegt kein Grund vor, die automatisch wirkenden Centren in die medulla spinalis zu verlegen, zusammenfallend mit den Kernen der die Athmungsmuskulatur versorgenden Nerven, für welche dann in der medulla oblongata ein regulirendes Centrum enthalten ist ebenso wie sich ja daselbst auch ein regulirendes Centrum für die Herzthätigkeit befindet. 